\title{
The mortality of MOP3 deficient mice with a systemic functional failure
}

\author{
Yimin Sun ${ }^{1}$, Zhihui Yang ${ }^{2}$, Zeqing Niu ${ }^{3}$, Wenle Wang ${ }^{4}$, Jianxiao Peng ${ }^{3}$, Qinghuan $\mathrm{Li}^{3}$, \\ Mark Y. Ma ${ }^{2, *} \&$ Yong Zhao ${ }^{1,3, *}$ \\ ${ }^{1}$ Department of Surgery, University of Nebrasaka Medical Center, Omaha, NE, 68131-5805, USA; \\ ${ }^{2}$ Department of Anatomy and Cell Biology, University of Nebraska Medical Center, Omaha, NE, 68131-5805, \\ USA; ${ }^{3}$ Transplantation Biology Research Division, The State Key Laboratory of Biomembrane and Mem- \\ brane Biotechnology, Institute of Zoology, Chinese Academy of Sciences, Beisihuan Xi Road 25, Haidian, \\ Beijing, 100080, China; ${ }^{4}$ Department of Pathology, Medical Center, University of Maryland, Baltimore, MD, \\ USA
}

Received 11 April 2006; accepted 28 July 2006

(c) 2006 National Science Council, Taipei

Key words: MOP3, life span, molecular clock, PAS

\begin{abstract}
Summary
MOP3 (also known as BMAL1), a master regulator of circadian rhythm, plays important roles in the regulation of cell differentiation and general physical functions. In the present studies, MOP3 deficient mice had significantly reduced body weight and showed remarkable mortality around six months of age. The levels of AST, ALT, BUN, or UREA in the blood of about four month-old MOP $3^{-/-}$mice were significantly higher than $\mathrm{MOP}^{+/-}$or MOP3 ${ }^{+/+}$littermates. However, no apparent pathological changes in the livers, hearts, lungs or kidneys of about four month-old $\mathrm{MOP}^{-/-}$mice were observed. In addition, altered levels of white blood cells, lympgocytes, and platelets in peripheral blood of $\mathrm{MOP} 3^{-/}$mice were detected. The results presented herein with MOP3-deficient mice offered the basic principle for the essential roles of MOP3 in keeping normal survival abilities in mice. This study may have significant clinical impacts on the consideration about the abnormality of circadian rhythms and sleeping disorders caused physical and metabolism dysfunctions as well as the mortality.
\end{abstract}

Abbreviations: ALT - alanine aminotransferase; ARNT - aryl hydrocarbon receptor nuclear translocator; AST - asparate aminotransferase; bHLH - basic-helix-loop-helix domain; BMAL1 - brain and muscle Arnt-like protein-1; CLOCK - product of the clock locus in mice; HIF - hypoxia-inducible factor; PAS PER-ARNT-SIM homology domain; SGPT - serum glutamic pyruvic transaminase; SGOT - serum glutamic oxalocetic transaminase

\section{Introduction}

The basic-helix-loop-helix (bHLH)-PER-ARNTSIM (PAS) superfamily plays crucial roles in adaptive responses to low atmospheric and cellular oxygen levels, exposure to certain environmental pollutants, and diurnal oscillations in light and temperature [1-3]. MOP3, also known as aryl

*To whom correspondence should be addressed. Fax: (86) 10-62659958; E-mail: zhaoy@ioz.ac.cn, yma@unmc.edu hydrocarbon receptor nuclear translocator 3 (ARNT3) or brain and muscle Arnt-like protein-1 (BMAL1), is a member of PAS superfamily, and a key transcription factor in the transcriptional/ translational feedback loop of mammalian circadian genes [4]. It was previously shown that mice deficient in the BMAL1/Mop3 gene become immediately arrhythmic in constant darkness and have reduced locomotor activity levels under entrained and constant conditions. MOP3 forms heterodimers with another bHLH/PAS protein, 
CLOCK (product of the clock locus in mice), which drive transcription from E-box elements found in the promoter of circadian responsive genes, including period 1 and cryptochrome [5].

Although the precise role of peripheral clocks and the mechanisms that link them to the hypothalamic suprachiasmatic nucleus remain largely obscure, the genetic techniques, including gene mutation or deletion, have indicated that some these genes might play a regulatory role in cellular function, including cell division, estrous and phospholipids metabolism [6]. Recent studies indicate that BMAL1, a master regulator of circadian rhythm, also plays important roles in the regulation of adipose differentiation and lipogenesis in mature adipocytes, the glucose homeostasis, and energy balance [7-9]. Here, our experimental results in MOP3 deficient mice have shown that MOP3 deficient mice died around six months of age with a serious dysfunction of livers, kidneys and hearts in these mice, whereas the MOP3 heterogeneous and wild-type mice lived well. The present study suggested that MOP3 might have much crucial physiological roles in mice in general.

\section{Materials and methods}

\section{Animals}

Homozygous MOP3 deficient mice $\left(\mathrm{MOP}^{-/-}\right)$in C57BL/6 background were produced by breeding heterozygous MOP3 deficient pairs $\left(\mathrm{MOP}^{+/-}\right)$ that were kindly provided by Dr. Christopher A. Bradfield, University of Wisconsin Medical School, Madison, WI. The expression of MOP3 was confirmed by RT-PCR as reported before [4]. All mice were maintained in a specific pathogenfree facility and were housed in microisolator cages containing autoclaved feed, bedding, and water. Animal care was in accordance with the American Association for the Accreditation of Laboratory Animal Care and institutional guidelines.

\section{Haematology assays}

Blood samples were collected into EDTA tubes for haematology analysis. Haematology was measured using a Roche ABX blood cell counter (Cobas Minos Vet, Roche Diagnostic Systems) and ABX Minoton LMG diluent.

\section{Blood Biochemistry analysis}

Blood biochemistry including Serum glucose, protein, cholesterol, creatinine and others was detected using a Conas Mira (Roche Diagnostic Systems).

Haematoxylin and eosin $(H-E)$ staining of tissues

The brain and peripheral tissues were used. Tissues were fixed in $10 \%$ buffered neutral formalin, embedded in paraffin wax and $4 \mathrm{~m}$ thick sections were cut and stained with $\mathrm{H}-\mathrm{E}$.

\section{Statistical analysis}

All data are reported as the mean \pm SD. Student's $t$ test for comparison of means was used to compare groups. A $p$ value less than 0.05 was considered to be statistically significant.

\section{Results and discussion}

The early Mortality and body weight loss of $\mathrm{MOP}^{-/-}$mice

Mice homozygous for the BMAL1/Mop3 deletion showed an attenuated rhythm of sleep and wakefulness distribution across the 24-h period. In addition, these mice showed increases in total sleep time, sleep fragmentation and EEG delta power under baseline conditions, and an attenuated compensatory response to acute sleep deprivation. Surprisingly, we found that mice deficient of MOP3 showed significantly decreased the body weight after three months of age (Figure 1A and B). The survival time of $\mathrm{MOP}^{-/-}$mice was about six months, which was markedly shorter than wildtype mice, in both male and female mice (Figure 1 $\mathrm{C}$ and D). As expected, the wild-type and heterogeneous mice lived well during observation period. In addition, the $\mathrm{MOP}^{-/-}$mice did not show any apparent clinical abnormalities in spire of the weight loss.

The blood biochemistry assays of $\mathrm{MOP}^{-/-}$mice

Brain and muscle Arnt-like protein-1 (BMAL1; also known as MOP3 or Arnt3) is a transcription factor known to regulate circadian rhythm. Its 

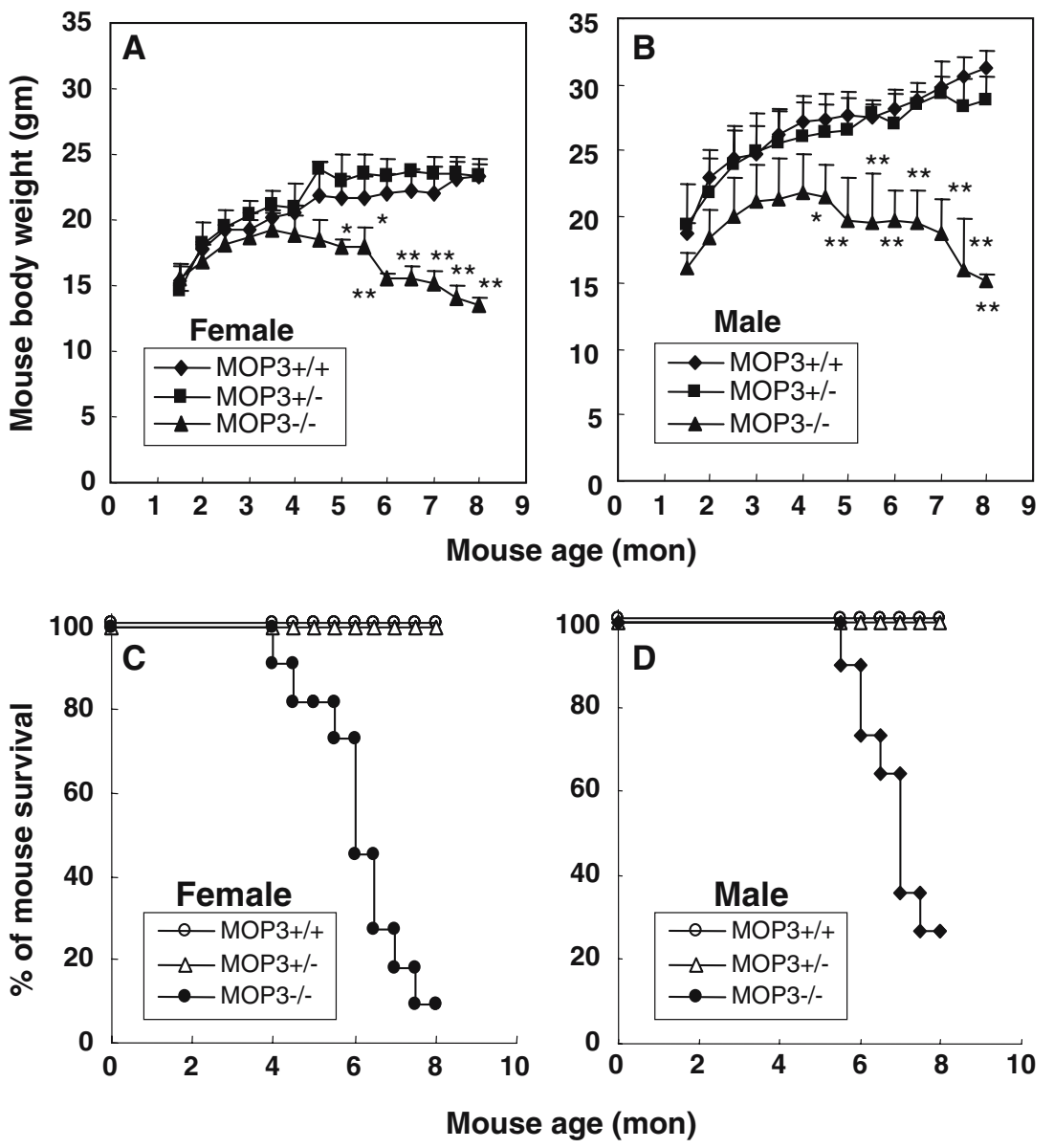

Figure 1. The survival and body weight changes of MOP3-deficient mice. The body weight of each mouse was recorded every two weeks and the mouse death was recorded daily. A summary of more than 15 mice in each group was shown. The body weight changes of female or male $\mathrm{MOP}^{-/-}$mice was shown in A and B, respectively. The survival of female and male MOP3 $3^{-/-}$mice was summarized in $\mathrm{C}$ and $\mathrm{D}$, respectively. ${ }^{*} p<0.05,{ }^{* *} p<0.01,{ }^{* * *} p<0.001$ compared with $\mathrm{MOP}^{+/-}$or $\mathrm{MOP}^{+/+}$mice. $^{*}$

involvement in the control of adipogenesis and lipid metabolism activity in mature adipocytes has been demonstrated. BMAL1 knockout mice embryonic fibroblast cells failed to be differentiated into adipocytes. Importantly, adding BMAL1 back by adenovirus gene transfer restored the ability of BMAL1 knockout mice embryonic fibroblast cells to differentiate. Furthermore, overexpression of BMAL1 in adipocytes increased lipid synthesis activity. These results indicate that BMAL1, a master regulator of circadian rhythm, also plays important roles in the regulation of adipose differentiation and lipogenesis in mature adipocytes.

As is shown in Figure 2, significantly biochemical changes of blood in $\mathrm{MOP}^{-/-}$mice that were about four months old were detected. Serum glutamic oxalocetic transaminase (SGOT) or asparate aminotransferase (AST) is an enzyme found primarily in the liver, heart, kidney, pancreas, and muscles. During tissue damages, especially in heart and liver, this enzyme is usually elevated. Serum glutamic pyruvic transaminase (SGPT) or alanine aminotransferase (ALT) is an enzyme found primarily in the liver but also to a lesser degree, the heart and other tissues. It is useful in diagnosing liver function more so than SGOT levels. Blood urea nitrogen (BUN) is the end product of protein metabolism and its concentration is influenced by the rate of excretion. Increased levels can be caused by excessive protein intake, kidney damage, liver damage, certain drugs, low fluid intake, intestinal bleeding, exercise or heart failure. Decreased levels may be due to a poor diet, liver 

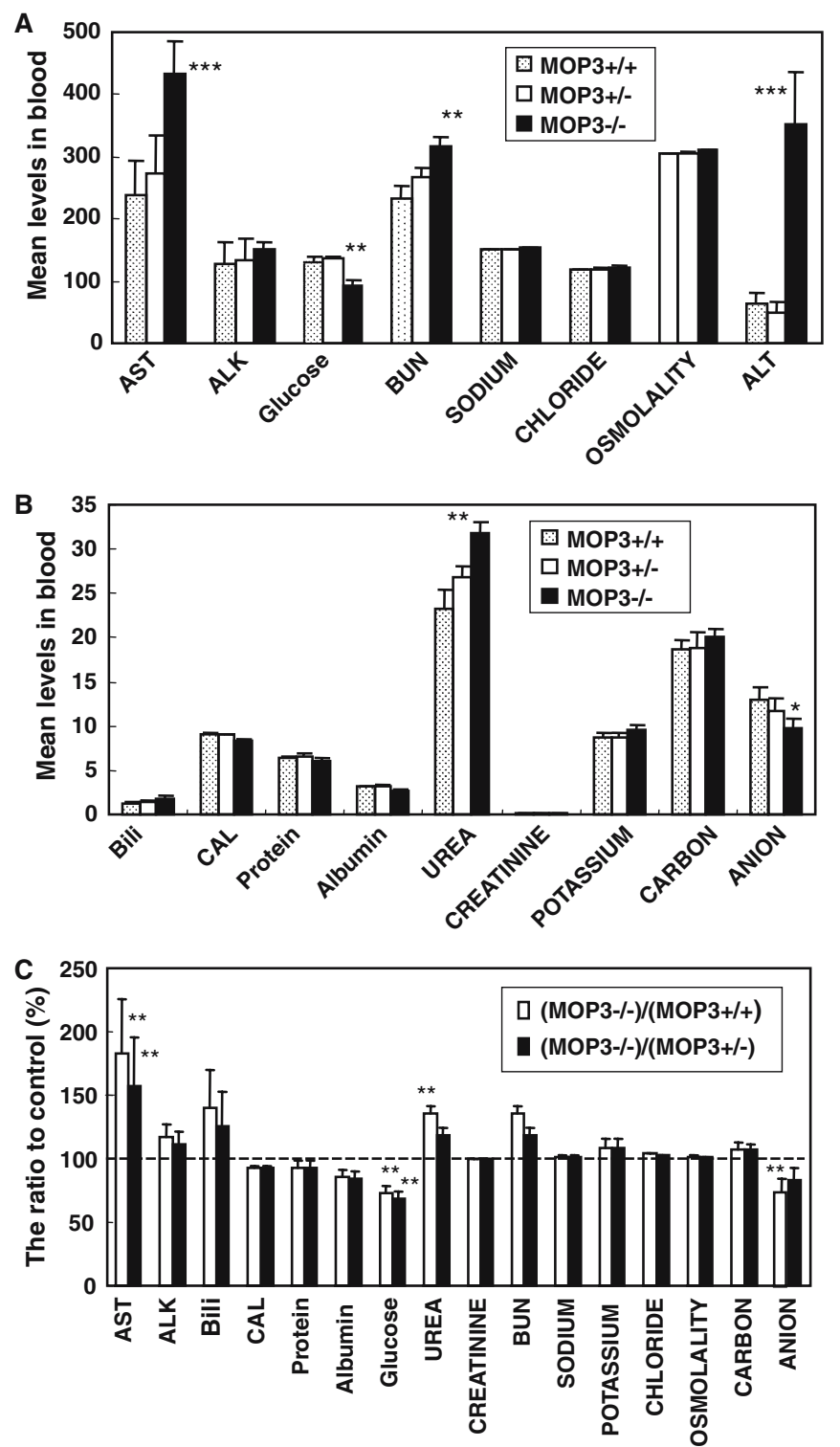

Figure 2. The blood biochemistry assays of $\mathrm{MOP}^{-/-}$or control mice. A summary of more than ten mice that were about four months old in each group was shown in $\mathrm{A}$ and $\mathrm{B}$. The ratios of $\mathrm{MOP}^{-/-}$to $\mathrm{MOP} 3^{+/+}$mice or $\mathrm{MOP} 3^{-/-}$to $\mathrm{MOP}^{+/-}$mice were shown in C. ${ }^{*} p<0.05,{ }^{* *} p<0.01,{ }^{* * *} p<0.001$ compared with $\mathrm{MOP}^{+/-}$or $\mathrm{MOP}^{+/+}$mice.

damage or low nitrogen intake. The strikingly increased levels of AST and ALT in the blood of $\mathrm{MOP}^{-/-}$mice indicated that the livers, hearts or kidneys of these mice might have serious functional abnormality. However, when the tissues were evaluated with $\mathrm{H}-\mathrm{E}$ staining, no significant pathological alterations were observed in these about four month-old MOP3 $3^{-/}$mice which showing identical tissue structures to control mice (Figure 3). Thus, the major changes in $\mathrm{MOP}^{-1-}$ mice may occur in the respects of function.
The anion gap is used to measure the concentration of cations (sodium and potassium) and the anions (chloride and $\mathrm{CO}_{2}$ ) in the extracellular fluid of the blood. An increased level is associated with metabolic acidosis due to the overproduction of acids (a state of alkalinity is in effect), whereas decreased levels may indicate metabolic alkalosis due to the overproduction of alkaloids (a state of acidosis is in effect). Glucose, formed by the digestion of carbohydrates and the conversion of glycogen by the liver is the primary source of energy 


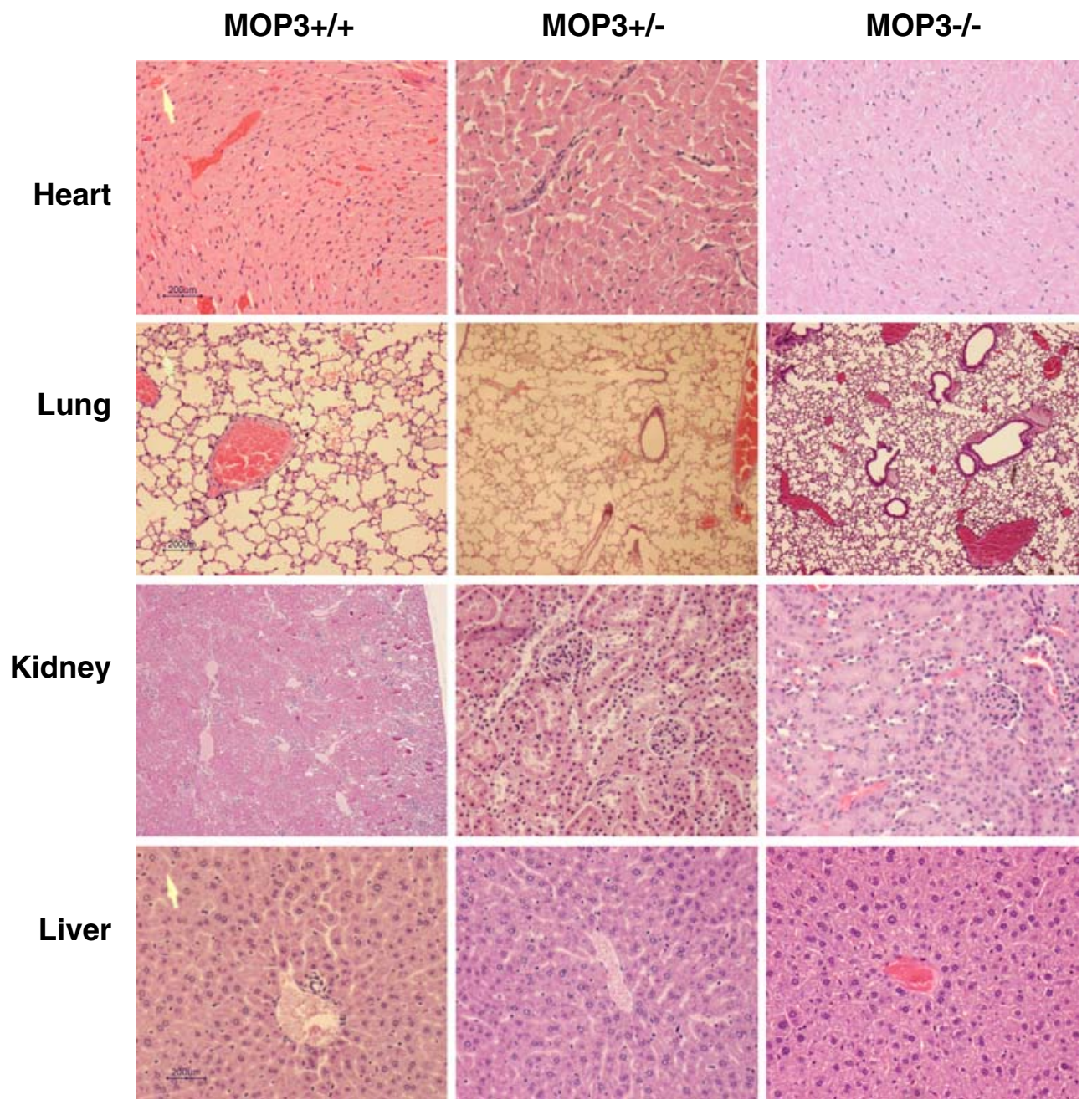

Figure 3. The $\mathrm{H}-\mathrm{E}$ staining of the livers, lungs, hearts and kidneys of MOP3 mice. Tissues from $\mathrm{MOP}^{-/-}, \mathrm{MOP}^{+/-}$or $\mathrm{MOP}^{+/+}$mice were stained with $\mathrm{H}-\mathrm{E}$. No significant differences among these mice were observed. More than four mice in each group were performed.

for most cells in bodies. It is tightly regulated by insulin, glucagon, thyroid hormone, liver enzymes and adrenal hormones. It is elevated in diabetes, liver disease, obesity, steroids, or stress. Low levels may be indicative of liver diseases, overproduction of insulin, or hypothyroidism. The significant changes of anion and glucose in the blood of $\mathrm{MOP}^{-/-}$mice suggested that the metabolic abnormality might present in these mice.

The altered levels of immune cells in $\mathrm{MOP}^{-/-}$mice

It has been reported that MOP3 mRNA expression overlaps in a number of tissues with each of MOP4, CLOCK, HIF $1 \alpha$ and HIF- $2 \alpha$, and that MOP 3 is a general dimerization partner for each of these four transcriptional regulators via a direct interaction. Transfection experiments have demonstrated that MOP3 formed transcriptionally active complexes with HIF $1 \alpha$ and HIF $2 \alpha$, and that these complexes responded to cellular hypoxia. MOP3/CLOCK complexes are shown to employ E-Box, which is a widely used DNA control element [5]. Despite its brevity and broad distribution, the E-Box is a remarkably versatile sequence that affects many different genetic programs, including proliferation, differentiation, tissue-specific responses, and cell death [10]. Hematopoiesis occurs under relatively hypoxic conditions in the bone marrow [11]. About 4-5 months old $\mathrm{MOP}^{-/}$mice showed signifi- 


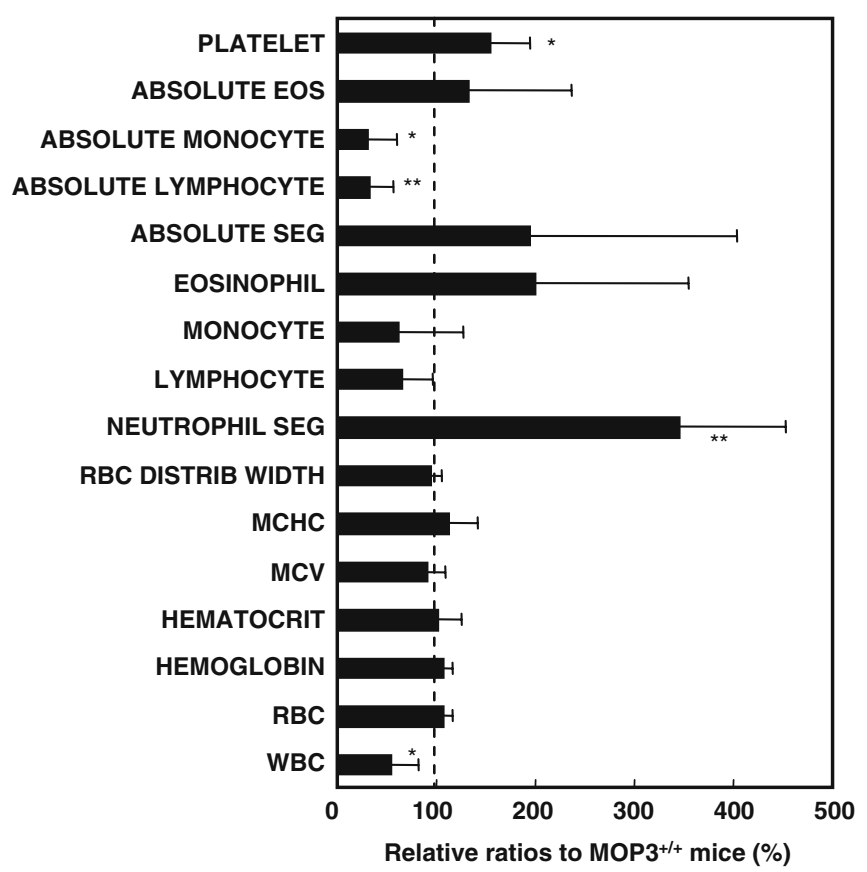

Figure 4. The altered levels of immune cells in the peripheral blood in MOP3-/- mice. About 4-5 months old mice were used in this study. ${ }^{*} p<0.05$ compared with $\mathrm{MOP} 3^{+/+}$mice. Eight mice in each group were performed.

cantly enhanced levels of segmented neutrophil and platelet as well as decreased levels of lymphocytes and white blood cells (WBCs)in the peripheral blood, compared with $\mathrm{MOP}^{+/-}$and $\mathrm{MOP}^{+/+}$ mice (Figure 4 and data not shown). In addition, significantly decreased levels of $\mathrm{B}$ cells but not $\mathrm{T}$ cells were observed in $\mathrm{MOP}^{-/-}$mice (data not shown). It seems that the mild changes in the immune system of $\mathrm{MOP}^{-/-}$mice might not be sufficient to explain the significantly enhanced motility of these mice.

Nevertheless, the results presented herein with MOP3 deficient mice offer the basic principle for the essential role of MOP3 in keeping normal survival abilities in mice. Significantly altered internal organs' function and metabolism disorders were observed in $\mathrm{MOP}^{-/-}$mice. This study may have significantly clinical impacts on the consideration about the abnormality of circadian rhythms and sleeping disorders caused physical and metabolism dysfunctions as well as the mortality.

\section{Acknowledgements}

We thank Dr. Guangwei Liu for his review of the manuscript. This work was supported by grants from the Knowledge Innovation Program of Chinese Academy of Sciences (KSCX2-SW-333), 100 Quality Vocational Colleagues of Chinese Academy of Sciences (2003), National Science Fund for Distinguished Young Scholar, National Natural Science Foundation of China (C03020504), the National Basic Research Program (973 Program, 2003CB515501), the Scientific Research Foundation for the Returned Overseas Chinese Scholars of State Education Ministry (2005-546, Y.Z.), and Nebraska Department of Health and Human Services.

\section{References}

1. Hankinson O., Brooks B.A., Weir-Brown K.I., Hoffman E.C., Johnson B.S., Nanthur J., Reyes H. and Watson A.J., Genetic and molecular analysis of the Ah receptor and of Cyplal gene expression. Biochimie. 73: 61-66, 1991.

2. Burbach K.M., Poland A. and Bradfield C.A., Cloning of the Ah-receptor cDNA reveals a distinctive ligand-activated transcription factor. Proc. Natl. Acad. Sci. USA 89: 8185-8189, 1992.

3. King D.P., Zhao Y., Sangoram A.M., Wilsbacher L.D., Tanaka M., Antoch M.P., Steeves T.D., Vitaterna M.H., Kornhauser J.M., Lowrey P.L. et al, Positional cloning of the mouse circadian clock gene. Cell 89: 641-653, 1997.

4. Bunger M.K., Wilsbacher L.D., Moran S.M., Clendenin C., Radcliffe L.A., Hogenesch J.B., Simon M.C., Takahashi 
J.S. and Bradfield C.A., Mop3 is an essential component of the master circadian pacemaker in mammals. Cell 103: 1009-1017, 2000.

5. Hogenesch J.B., Gu Y.Z., Jain S. and Bradfield C.A., The basic-helix-loop-helix-PAS orphan MOP3 forms transcriptionally active complexes with circadian and hypoxia factors. Proc. Natl. Acad. Sci. USA 95: 5474-5479, 1998.

6. Matsuo T., Yamaguchi S., Mitsui S., Emi A., Shimoda F. and Okamura H., Control mechanism of the circadian clock for timing of cell division in vivo. Science 302: 255 259, 2003.

7. Shimba S., Ishii N., Ohta Y., Ohno T., Watabe Y., Hayashi M., Wada T., Aoyagi T. and Tezuka M., Brain and muscle Arnt-like protein-1 (BMAL1), a component of the molecular clock, regulates adipogenesis. Proc. Natl. Acad. Sci. USA 102: 12071-12076, 2005.
8. Rudic R.D., McNamara P, Curtis A.M., Boston R.C., Panda S., Hogenesch J.B. and Fitzgerald G.A., BMAL1 and CLOCK, two essential components of the circadian clock, are involved in glucose homeostasis. PLoS. Biol. 2: 1893-1899, 2004.

9. Turek F.W., Joshu C., Kohsaka A., Lin E., Ivanova G., McDearmon E., Laposky A., Losee-Olson S., Easton A., Jensen D.R. et al, Obesity and metabolic syndrome in circadian Clock mutant mice. Science 308: 1043-1045, 2005.

10. Munoz E., Brewer M. and Baler R., Circadian Transcription. Thinking outside the E-Box. J. Biol. Chem. 277: 36009-36017, 2002.

11. Pennathur-Das R. and Levitt L., Augmentation of in vitro human marrow erythropoiesis under physiological oxygen tensions is mediated by monocytes and $\mathrm{T}$ lymphocytes. Blood 69: 899-907, 1987. 